\title{
Research on Vehicle Positioning Method Based on Underground Garages
}

\author{
Min Liu ${ }^{1, a, *}$, Suli $\mathrm{He}^{1}$ and Ruibin Liu ${ }^{1}$ \\ ${ }^{1}$ South China Institute of Software Engineering GU, Department of Electronic Studies, 510990, Guangzhou, China
}

\begin{abstract}
With the continuous development of urbanization, the number of vehicles has increased year by year. In order to solve the hard-to-park problem, underground garages have been built on a large scale in many cities. However, due to the fact that GPS signals are kept out in the underground garage, GPS technology does not work. As a consequence, drivers cannot use navigation equipment to search for the parking space. In view of this, vehicle positioning technique for vehicles in underground garages has hold the public attention and relevant research has been done. In this paper, we propose an efficient method of accurate vehicle positioning. First, use the low-power Bluetooth device, then use Gaussian filter to optimize the RSSI ranging algorithm, and then use the maximum likelihood method to improve the three-loop positioning algorithm. Finally, a comparison is made between the algorithm and the traditional algorithm. A large number of experiments have proved that this method can be used to determine the positions of vehicles in underground garages.
\end{abstract}

\section{Introduction}

AIn outdoor environment, GPS, the most successful and widely used positioning technique, may satisfy most needs of its users ${ }^{[1-2]}$. However, in an underground garages, buildings and walls may absorb microwave. Moreover, the underground garage is featured by complex indoor communication channel. As a result, GPS signals are characteristic of large signal attenuation and measuring errors. Worse enough, positioning fails when signals are obstructed. In view of this, GPS positioning technique is not applicable in underground garages $^{[3-4]}$.What's more, Bluetooth Low Energy (BLE), a new bluetooth technology, has many advantages: low power consumption, low time delay and low handling capacity. Besides, one button battery may work for years in a row. For this reason, BLE has become the first choice among short distance wireless communication techniques for smart mobile terminal, attracting the attention of the business circles and research scholars. The wireless signals of BLE technique has RSSI value, and RSSI-based positioning algorithm needs no extra hardware. Besides, comparatively, RSSI-based rangebased positioning algorithm boasts higher precision than the positioning algorithm which needs no ranging. For this reason, BLE technique has become a research highlight concerning vehicle positioning in underground garages $^{[5]}$.

\section{Optimization of RSSI-based Indoor Ranging Technology}

In the final analysis, vehicle positioning in underground garages belongs to indoor positioning. For indoor distance measurement, what is mostly used is wireless sensor-based RSSI ranging technology. The literature [6] analyzes the undesirable effects of various elements in real indoor environment (barriers, long-distance transmission and weather, etc) on wireless signal transmission, resulting in collected RSSI data exception. Because of this, how to filter the collected RSSI data, i.e, to filter the anomalous RSSL data caused by burst interference and random disturbance, is a challenge to be addressed in indoor ranging. Moreover, another key factor concerning RSSL-based indoor ranging technique is the selection of location models and parameter optimization in the model. Literatures [7] and [8] mention that satisfying result has been achieved to optimize the model parameter using linear regression algorithm. Based on above-mentioned factors, this system proposes indoor range-measurement system based on low power consumption bluetooth. By analyzing the existing RSSI data processing algorithm, a new RSSI filtering algorithm is put forward patulous Gaussian filter. In addition, maximum likelihood method is used to improve the three-loop positioning algorithm. 


\subsection{RSSI Value Optimization Based on Patulous Gaussian Filter ${ }^{[9]}$}

Gaussian filter has found wide application in that it can effectively minimize the effects on RSSI value caused by short-time disturbance or disturbances resulting from emergency. However, nonparoxysmal disturbances such as multipath attenuation, can still affect the accuracy of test values. We may assume that the whole RSSI values received accord with Gaussian distribution. However, as a matter of fact, there exist different mean values and variances between the values affected by multipath attenuation and the sound received signal values. If the former and the sound signal values are taken as the whole Gaussian distribution for filtering, the result thus obtained is not accurate. Therefore, this paper proposes patulous Gaussian filter method: split the whole received RSSI values into combinations of different Gaussian distributions of good values and defective values so as to filtrate the RSSI values affected by the multipath attenuation signals. Next, further filter and optimize these RSSL values to increase the ranging accuracy and precision of positioning.

Patulous Gaussian filter means splitting the observed data values into several combinations of Gaussian probability-density functions representative of normal distribution curve. The probability density functions are as shown below:

$$
\begin{gathered}
P(x)=\sum_{k=1}^{k} W_{k} N\left(X ; U_{K}, \sigma_{k}^{2}\right) \\
W_{K}=\frac{N_{K}}{N}
\end{gathered}
$$

$\mathrm{K}$ is the number of groups of probability density functions, $\left(X ; U_{k^{\prime}} \sigma_{k}^{2}\right)$ is the distribution function of one component, which is obtained from its RSSL value. $\mathrm{W}_{\mathrm{k}}$ is weighing factor, $\mathrm{N}_{\mathrm{k}}$ is the number of RSSI values of the components in the kth group, $\mathrm{N}$ is the number of all RSSI values, $U_{k}$ is the mean value of the components in the kth group, and $\sigma_{k}^{2}$ is the variance of the components in the kth group. may refer to the stable values among the RSSI values in the kth group. Among the signals in the kth group, one group is direct signal group, i.e, ideal signal group while the components of the other K-1 groups are viewed as multipath attenuation signals. Therefore, after the RSSL values of multipath attenuation signals are separated, the RSSI value of the ideal signals may be obtained, that is, the mean value of the ideal signal group.

In the process of clustering the patulous Gaussian filter, the known quantity is the received RSSI value data while the parameters of the probability density functions of the groups are unknown. Therefore, the unknown parameter may be obtained through expectationmaximization algorithm. Expectation-maximization algorithm is a method by which maximum likelihood estimation of the parameter is acquired through iteration method. The following is the algorithmic principle:

Let be the $\mathrm{W}_{\mathrm{k}}$ selected probability in the kth group. Introduce a new random variable $\mathrm{b} . \mathrm{b}$ is $\mathrm{k}$ dimension. Each component in $b$ is $b_{k}(k=1,2, \ldots \ldots ., k)$ can only get two values: 0 or 1 . When $b_{k}=1$, it means the kth group is selected. Conversely, if $b_{k}=0$, it means the kth group fails to be selected. From this, it is clear that the selected probability of the kth group is the probability that the kth dimension component of $\mathrm{b}$ is 1 , namely:

$$
W_{k}=p(k=1)
$$

In this formula, $b_{k}$ should satisfy the following condition:

$$
b_{k} \in\{0,1\}, \sum_{k=1}^{k} b_{k}=1
$$

The above-mentioned formula means that when we choose the kth group, grouping has been done. Therefore, apart from the kth dimension of $b$, its other components are 0 , and the sum of the probabilities of other groups is 1 , hence satisfying the normalizing condition of probability. As we have no special conditions for grouping selection, we may assume that b's components are independent identically distributed, hence acquiring joint probability distribution of $\mathrm{b}$ :

$$
P(b)=p(b 1) p(b 1) \ldots p(b 1)=\prod_{k=1}^{k} w_{k}^{b_{k}}
$$

Also, as the data in the groups are still subject to Gaussian distribution, after we have chosen the subgroup, the probability density function acquired is the probability density function of the group's Gaussian distribution. This means the distribution that $\mathrm{x}$ satisfies when $b_{k}=1$. The contingent probability is described as follows:

$$
P(X \mid b)=\prod_{k=1}^{k} N\left(X ; \mu_{k^{\prime}} \sigma^{2}\right)^{b_{k}}
$$

Based on formula 5 and formula 6 , we may get the known b's probability and the probability of $\mathrm{x}$ when satisfying condition $b$. Based on formula of total probability, we can acquire $x$ 's probability:

$$
P(X)=\sum_{\mathrm{b}} P(b) P(X \mid b)
$$

Put formula 5 and formula 6 into formula 7 , and have it arranged, and we obtain:

$$
P(X)=\sum_{k=1}^{k} W_{k} N\left(X ; \mu_{k^{\prime}} \sigma_{k}^{2}\right)
$$

It can be seen that in Formulas 8 , we introduce implicit viable $\mathrm{b}$ of $\mathrm{K}$ dimension. It is aimed at obtaining the posterior probability of $\mathrm{x}$ belonging to the kth group when we have detected the datum $\mathrm{x}$.

According to Bayesian formula, we know that $\mathrm{p}(\mathrm{b})$ is prior probability, and $\mathrm{p}(\mathrm{x} \mid \mathbf{b})$ is contingent probability. Therefore, posterior profitability may be acquired $\mathrm{p}(\mathrm{z} \mid \mathbf{x})$. It means that when we obtain the observed valued $x$ (RSSI value), the probability of value $x$ belonging to the kth group $\left(b_{k}=1\right)$ is:

$$
P\left(b_{k}=1 \mid X\right)=\frac{P(X \mid b=1) P\left(b_{k}=1\right)}{P(X)}
$$

Put formula 3, formula 6-10 and formula 8 respectively into the above-mentioned formula, and have it arranged, then we get:

$$
P\left(b_{k}=1 \mid X\right)=\frac{W_{k} N\left(X ; \mu_{k}, \sigma_{k}^{2}\right)}{\sum_{k}^{i=1} W_{i} N\left(X ; \mu_{i} \sigma_{i}^{2}\right)}
$$

With the experience we get in doing experiment, generally, the acquired RSSI values may be divided into three groups, namely, the ideal signal set and two multipath attenuation signal groups. The values of other signal groups are too small to be considered. Therefore, in this model, K's value is often 2 or 3 . When $\mathrm{k}=1$, it is ordinary Gaussian filter. When $\mathrm{k}=2$ or $\mathrm{k}=3$, it is shown as following:

When $\mathrm{k}=2$, the RSSI values collected are divided into two constituent parts. N RSSI values are respectively. 
Definition $\mathrm{P}_{1}\left(\mathrm{RSSI}_{\mathrm{i}}\right)$ means RSSI $\mathrm{I}_{\mathrm{i}}$ belongs to the probability of the first group, i.e, probability of the ideal group. It is known from formula (3-14) that:

$$
P_{1}\left(R S I_{i}\right)=\frac{W_{1} f\left(R S I_{i} ; \mu_{1}, \sigma_{1}^{2}\right)}{W_{1} f\left(R S S I_{i} ; \mu_{1}, \sigma_{1}^{2}\right)+W_{2} f\left(R S I_{i} ; \mu_{2}, \sigma_{2}^{2}\right)}
$$

$\mathrm{P}_{2}\left(\mathrm{RSSI}_{\mathrm{i}}\right)$ means RSSI $\mathrm{R}_{\mathrm{i}}$ belongs to the probability of the second group:

$$
P_{2}\left(R S S I_{i}\right)=\frac{W_{2} f\left(R S I_{i} ; \mu_{2}, \sigma_{2}^{2}\right)}{W_{1} f\left(R S I_{i} ; \mu_{1}, \sigma_{1}^{2}\right)+W_{2} f\left(R S I_{i} ; \mu_{2}, \sigma_{2}^{2}\right)}
$$

After calculation, probability density function and the values of parameters may be acquired, hence probabilities of different groups. If $\mathrm{P}_{1}\left(\mathrm{RSSI}_{\mathrm{i}}\right)$ value is larger than that of $\mathrm{P}_{2}\left(\mathrm{RSSI}_{\mathrm{i}}\right)$, then the RSSI value belongs to the first group, i.e, the ideal group. Otherwise, it belongs to the second group, i.e, multipath attenuation group.

Similarly, when $\mathrm{K}=3$, RSSI value is split into one ideal group and two multipath attenuation groups. Use to represent the probabilities of the ideal group, the first multipath attenuation group and the second multipath attenuation group respectively, as shown below:

$$
\begin{aligned}
& P_{2}\left(R S S_{l}\right)=\frac{W_{1} f\left(R S S_{i} ; \mu_{1}, \sigma_{1}^{2}\right)}{W_{1} f\left(R S S \zeta ; \mu_{1}, \sigma_{1}^{2}\right)+W_{2} f\left(R S S \zeta ; \mu_{2}, \sigma_{2}^{2}\right)+W_{3} f\left(R S S I ; \mu_{3}, \sigma_{3}^{2}\right)} \\
& P_{2}\left(R S S l_{i}\right)=\frac{W_{2} f\left(R S S \xi ; \mu_{2}, \sigma_{2}^{2}\right)}{W_{1} f\left(R S S \xi_{i} ; \mu_{1}, \sigma_{1}^{2}\right)+W_{2} f\left(R S S ; ; \mu_{2}, \sigma_{2}^{2}\right)+W_{3} f\left(R S S \xi ; \mu_{3}, \sigma_{3}^{2}\right)} \\
& P_{2}\left(R S S I_{i}\right)=\frac{W_{3} f\left(R S S i_{i} ; \mu_{3}, \sigma_{3}^{2}\right)}{W_{1} f\left(R S S I_{i} ; \mu_{1}, \sigma_{1}^{2}\right)+W_{2} f\left(R S S I_{i} ; \mu_{2}, \sigma_{2}^{2}\right)+W_{3} f\left(R S S I ; \mu_{3}, \sigma_{3}^{2}\right)}
\end{aligned}
$$

That is to say, when we want to divide $\mathrm{n}$ RSSL values into k groups, with the values of initial parameters $\mathrm{w}, \mu$ and $\delta^{2}$ preset, we may calculate the posterior probability of each RSSI belonging to the kth group. In it, $\mathrm{k}=1,2, \ldots \ldots, \mathrm{K}$. From probability normalization, we may know that:

$$
\sum_{k=1}^{k} P_{k}\left(R S S I_{i}\right)=1
$$

With N RSSI measured values, the sum of probabilities of all components belonging to the constituent parts is a fixed value $\mathrm{N}$. Then, the ratio of the sum of probabilities of the newly calculated different components belonging to the kth part to the total probability may be taken as the new weight value of the kth part. So, renew the weight value of the kth part to:

$$
W_{k}=\sum_{i=1}^{N} P_{k}\left(R S S I_{i}\right)
$$

Likewise, we renew the mean value and variance of the Gaussian distribution of the kth part to:

$$
\begin{gathered}
\mu_{k}=\frac{\sum_{i=1}^{N} P_{k}\left(R S S I_{i}\right) R S S I_{i}}{\sum_{i=1}^{N} P_{k}\left(R S S I_{i}\right)} \\
\sigma_{k}^{2}=\frac{\sum_{i=1}^{N} P_{k}\left(R S S I_{i}\right)\left(R S S I_{i}-\mu_{k}\right)^{2}}{\sum_{i=1}^{N} P_{k}\left(R S S I_{i}\right)}
\end{gathered}
$$

Repeat the iteration for the above-mentioned process until the change is smaller than the set threshold value. The final result is the probability of each RSSI value belonging to each group. Select those groups with larger probabilities as the final groups, and these RSSI values are divided into $\mathrm{k}$ groups. After grouping, compare the mean values and variances of each group so as to determine the ideal group and multipath attenuation group. Then, give up multipath attenuation component group, and retain the mean value of the ideal group as the chosen RSSI value. In this way, RSSI value is purified and optimized, hence enhancing the location precision.

\subsection{Three-loop Positioning Algorithm and Its Optimization}

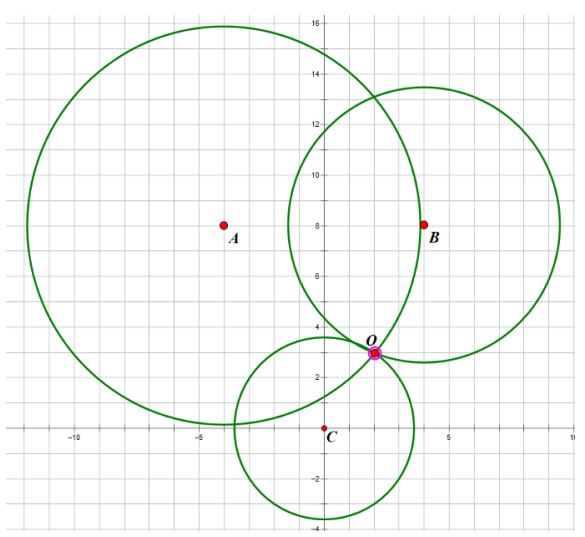

Fig.1 Schematic Diagram of Three-loop Positioning

The specific principle of three-loop positioning algorithm $^{[10]}$ : Let the sought point coordinate be $(\mathrm{x}, \mathrm{y})$. By measuring and calculating the distances between the three known coordinate points and the point: $d_{1}, d_{2}, d_{3}$, the equations may be written like this:

$$
\left\{\begin{array}{l}
\left(x_{1}-x\right)^{2}+\left(y_{1}-y\right)^{2}=d_{1}^{2} \\
\left(x_{2}-x\right)^{2}+\left(y_{2}-y\right)^{2}=d_{2}^{2} \\
\left(x_{3}-x\right)^{2}+\left(y_{3}-y\right)^{2}=d_{3}^{2}
\end{array}\right.
$$

In the equations, the coordinates of the three anchor nodes are respectively $\left(\mathrm{x}_{1}, \mathrm{y}_{1}\right),\left(\mathrm{x}_{2}, \mathrm{y}_{2}\right),\left(\mathrm{x}_{3}, \mathrm{y}_{3}\right)$. Through this equation set, the coordinate values $\mathrm{x}$ and $\mathrm{y}$ of the goal nodes may be solved.

In the actual process of positioning, due to the impact of measuring errors, multipath attenuation and stumbling blocks, the circle, with received base station coordinate as center, and measured distance as radius, more often than not, cannot meet at a point. This means that with the equation set insoluble, the centroid of the intersecting area is often taken as the final location coordinate of three-loop positioning algorithm.

As three-loop positioning algorithm has many flaws, some optimized algorithms based on three-loop positioning algorithm have been put forward from different perspectives. As shown in Fig 2, suppose that our smartphones receive signals from $\mathrm{n}$ base stations, and the coordinates of the base stations are known, the distances between the mobile device and the $\mathrm{n}$ base stations may be calculated according to the path loss models of the signals. Therefore, n-1 two-variable linear equations may be set up, hence system of linear equations. 


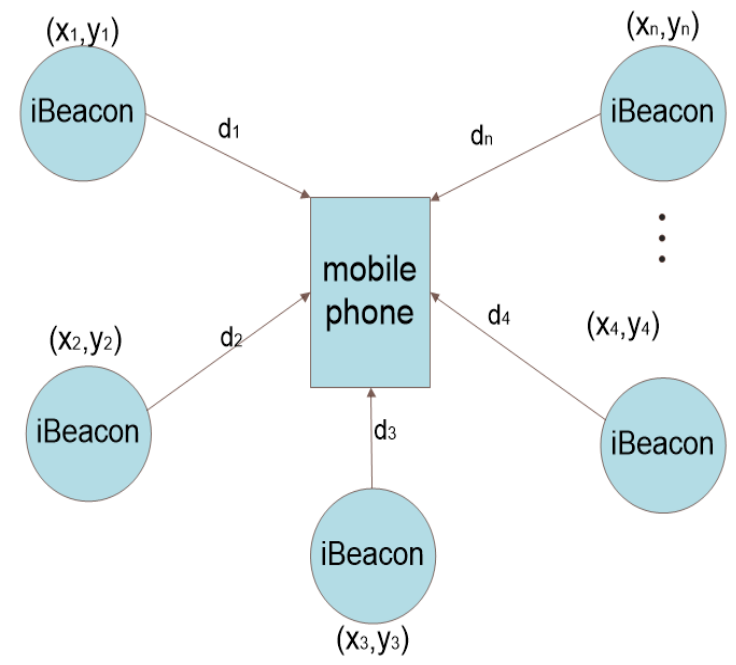

Fig. 2 Sketch map of maximum likelihood estimate method

The matrix representation of its equation set is: $\mathrm{AX}=\mathrm{b}$. In the matrix,

$$
A=\left[\begin{array}{cc}
2\left(x_{1}-x_{n}\right) & 2\left(y_{1}-y_{n}\right) \\
\cdots & \cdots \\
2\left(x_{n-1}-x_{n}\right) & 2\left(y_{n-1}-y_{n}\right)
\end{array}\right]
$$

$$
b=\left[\begin{array}{c}
x_{1}^{2}-x_{n}^{2}+y_{1}^{2}-y_{n}^{2}+d_{n}^{2}-d_{1}^{2} \\
\cdots \\
x_{n-1}^{2}-x_{n}^{2}+y_{n-1}^{2}-y_{n}^{2}+d_{n}^{2}-d_{n-1}^{2}
\end{array}\right]
$$

After calculation, the coordinate at the mobile phone is acquired: $\left(\mathrm{A}^{\mathrm{T}} \mathrm{A}\right)^{-1} \mathrm{~A}^{\mathrm{T}} \mathrm{b}$. In it, $\mathrm{A}^{\mathrm{T}}$ is $\mathrm{A}^{\prime}$ transposed matrix.

When the linear system of equations of the abovementioned maximum likelihood estimation is insoluble, its optimal solution is taken as estimated coordinate of the mobile terminal. The method to get the minimum error is least square method. In actual use, least square method and method of maximum likelihood are used in combination to solve the equation ${ }^{[11]}$.

\section{Experiment Comparison and Analysis}

In this test, the Bluetooth devices are put at three different places of the parking lot. As shown in Fig 3, the specific coordinates are: $\mathrm{A}(-80,160), \mathrm{B}(80,160)$, and $\mathrm{C}(0,0)$. The actual coordinate of the central point of the parking spaces is shown in Table 1.

Table 1. Actual coordinate of the central point of the parking spaces

\begin{tabular}{|c|c|c|c|c|c|c|}
\hline $\begin{array}{c}\text { Parking } \\
\text { Space }\end{array}$ & No.1/cm & No.2/cm & No.3/cm & No.4/cm & No.5/cm & No.6/cm \\
\hline $\begin{array}{c}\text { Actual } \\
\text { coordinates }\end{array}$ & $(320,182)$ & $(320,313)$ & $(320,546)$ & $(-320,182)$ & $(-320,313)$ & $(-320,546)$ \\
\hline
\end{tabular}

Table 2. A GT and survey coordinate of parking space using our method

\begin{tabular}{|c|c|c|c|c|c|c|}
\hline Method & No.1/cm & No.2/cm & No.3/cm & No.4/cm & No.5/cm & No.6/cm \\
\hline GT & $(331,488)$ & $(326,576)$ & $(339,846)$ & $(-328,486)$ & $(-323,572)$ & $(-341,842)$ \\
\hline Ours & $(311,459)$ & $(319,544)$ & $(327,825)$ & $(-317,455)$ & $(-309,557)$ & $(-337,817)$ \\
\hline
\end{tabular}

Table3. A comparison of errors between GT and out method

\begin{tabular}{|c|c|c|c|c|c|c|}
\hline Method & No.1/cm & No.2/cm & No.3/cm & No.4/cm & No.5/cm & No.6/cm \\
\hline GT & 306 & 263 & 301 & 304 & 259 & 297 \\
\hline Ours & 277 & 231 & 279 & 273 & 244 & 272 \\
\hline
\end{tabular}




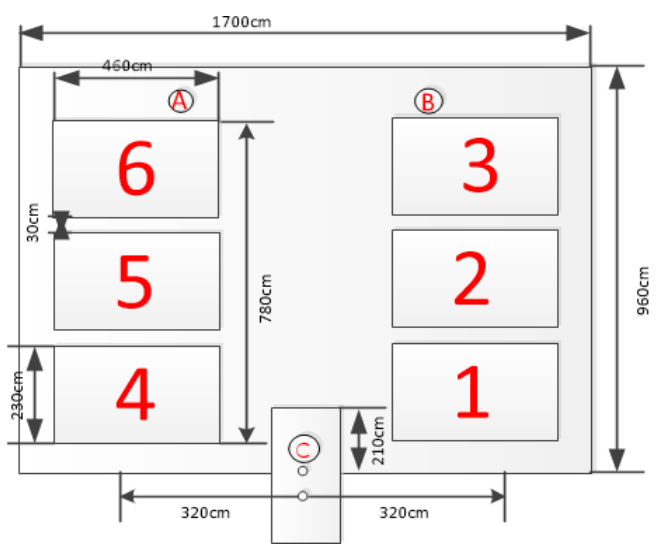

Fig.3 Overlooking planar graph of parking spaces

We may also use Gaussian filtering-Trilateral positioning (GT). A comparison experiment is done at the same underground garage, and experiment effect is shown in Table 2 and Table 3. It is clear that under the same condition, the error is much smaller if our method is used. It is true that in actual navigation, an error of one to three meters is permissible. However, a smaller error may enhance the performance of navigation. In this sense, compared with the traditional method, ours helps enhance the performance of navigation.

We may also use Gaussian filtering-Trilateral positioning (GT). A comparison experiment is done at the same underground garage, and experiment effect is shown in Table 2 and Table 3. It is clear that under the same condition, the error is much smaller if our method is used. It is true that in actual navigation, an error of one to three meters is permissible. However, a smaller error may enhance the performance of navigation. In this sense, compared with the traditional method, ours helps enhance the performance of navigation.

\section{Conclusion}

We propose an efficient and accurate vehicle positioning method. Ours is a method optimized on the basis of traditional location method. By our method, it means using Bluetooth with low power consumption as beacon to enhance the stand-by time of the system to the greatest extent. Through patulous Gaussian filtering, the whole received RSSI values are split into combinations of different Gaussian distributions of good values and bad values so as to filtrate the RSSI values affected by multipath weak signals. Then, by further filtrating and optimizing RSSI values, the accuracy of ranging is improved and positioning precision is enhanced. Least square method and maximum likelihood method are used in combination to optimize trilateral location algorithm. Experiments have shown that this method is superior to the traditional positioning method in that it not only helps to reduce the positioning error but also enhances the performance of navigation. To sum up, this method can be used to determine the positions of vehicles in underground garages.

\section{Fund project}

Foundation for Young Innovative Talents in Higher Education of Guangdong, China (No. 2020KQNCX139, 2019KQNCX221).

\section{References}

1. Kaplan E D, Hegarty C. Understanding GPS: principles and applications[M]. Artech House, 2005.

2. Shijun T, Yiming P. Research of weak GPS signal acquisition algorithm[C]. 2008 International Conference on Communications, Circuits and Systems. 2008: 793-796.

3. N. L D, F. G, P. Z. Wi Fi fingerprint indoor positioning system using probability distribution comparison[C].2012 IEEE International Conference on Acoustics, Speech and Signal Processing (ICASSP). 2012:2301-2304.

4. P. B, V. N P. RADAR: an in-building RF-based user location and tracking system[C]. INFOCOM 2000. Nineteenth Annual Joint Conference of the IEEE Computer and Communications Societies. Proceedings. IEEE. 2000: 775-784.

5. Research on Elaborate Indoor Positioning Method Based on iBeacon Firmware [J]. Zhang Zhuosheng, Ma Fangfang, Xue Jingyuan \& Ai Haojun, Geomatics World, 2015(02).

6. HU Bing, PENG Huijun, SUN Zhixin. LANDMARC Localization Algorithm Based on Weight Optimization[J]. Chinese Journal of Electronics, 2018, 27(06): 1291-1296

7. Mao W, He J, Qiu L. CAT: high-precision acoustic motion tracking[C] the 22nd AnnualInternational Conference. ACM, New York City,2016,69-81

8. Ni L M, Liu Y, Lau Y, et al. LANDMARC: Indoor location sensing using active RFID $[J]$. IEEE International Conference on Pervasive Computing \& Communications. 2003, 407

9. Zhang Rui, Research on Indoor Location Algorithm Improvement Based on iBeacon Firmware [D]. University of Electronic Science and Technology of China, 2019.

10. B. L, Z. W, J. C, et al. Research on the node location algorithm in the wireless sensor network[C].2011 International Conference on Electrical and Control Engineering. 2011: 350-353.

11. Luo Lanhua, Lianghaiying, \& Ren Ziting. Overview of the Node Localization Algorithm Based on Ranging of Wireless Sensor of Networks [J]. Science \& Technology View, 2016(3): 27-28 